\title{
Correction to: Blood Pressure-Lowering Therapy
}

\author{
Isabella Sudano (i), Elena Osto (1), and Frank Ruschitzka
}

\section{Correction to: \\ Chapter "Blood Pressure-Lowering Therapy" in: Isabella Sudano et al., Handbook of Experimental Pharmacology, https://doi.org/10.1007/164_2020_372}

The Open Access chapter 'Blood Pressure-Lowering Therapy' was published online unfortunately without the Conflict of Interest statement. The COI statement should appear as:

FR reports grant support for the ESC-HFA Postgraduate Course in Heart Failure from Novartis, Servier, Bayer, Abbott and Astra Zeneca and the VASCEND trial from Novartis (all payments directly to the University of Zurich).

FR has been paid for the time spent as a committee member for clinical trials, advisory boards, other forms of consulting and lectures or presentations. These payments were made directly to the University of Zurich and no personal payments were received in relation to these trials or other activities since January 2018.

The updated online version of this chapter can be found at https://doi.org/10.1007/164_2020_372

I. Sudano $\cdot$ F. Ruschitzka $(\bowtie)$

Department of Cardiology, University Heart Center Zurich, Zürich, Switzerland e-mail: frank.ruschitzka@usz.ch

E. Osto

Department of Cardiology, University Heart Center Zurich, Zürich, Switzerland

Institute of Clinical Chemistry, University of Zurich, University Hospital Zurich, Zürich, Switzerland 
Before 2018, FR reports grants and personal fees from SJM/Abbott, Servier, Novartis and Bayer, personal fees from Zoll, Astra Zeneca, Sanofi, Amgen, BMS, Pfizer, Fresenius, Vifor, Roche, Cardiorentis and Boehringer Ingelheim, other from Heartware and grants from Mars, outside the submitted work.

These corrections have been updated in the original chapter. 